\title{
Arbeitsgestaltung im demografischen Wandel - Bilanzierung des Fortschritts von 1999 bis 2019
}

\author{
T. Langhoff
}

\section{Abstract}

Arbeitsgestaltung ist durch den demografischen Wandel zu einem Wettbewerbsfaktor geworden. Eingangs analysiert der Beitrag die Auswirkungen auf Alterung der Belegschaften und Rekrutierung im zeitlichen Verlauf. Anschließend werden Meilensteine der Diffusion arbeits- und personalwissenschaftlicher Erkenntnisse der vergangenen 20 Jahre in die betriebliche Praxis vorgestellt. Eine zentrale Zukunftsaufgabe bleibt der stärkere Transfer dieser Erkenntnisse zu betrieblichen Führungskräften.

\section{Einleitung}

Im Jahr 2020 haben Themen wie Globalisierung, Digitalisierung, Klimawandel und natürlich der Umgang der Corona Pandemie den demografischen Wandel als zentrales und ubiquitäres Thema der letzten beiden Jahrzehnte fast schon abgelöst. Zumindest hat die betriebliche Praxis verstanden, die neu aufkommenden Themen immer auch mit der demografischen Brille zu betrachten, denn eine relative Gleichverteilung der Altersgruppen in den Betrieben wird sich erst ca. ab 2035 einstellen, wenn die Alterskohorte der Babyboomer (Geburten zwischen 1955 und 1965) endgültig in die Rente abgewandert ist, und die Geburtenrate von 1,5 Kinder je Frau, die ab 1970 in etwa konstant ist, wirkt. Die permanente Schrumpfung unserer Gesellschaft hält weiter an.

Das Thema demografischer Wandel und insbesondere die Alterung vieler Belegschaften bleibt akut und man kann sagen, dass Arbeitswissenschaft und betriebliche Praxis das Altern im Blick bzw. verinnerlicht haben. Dazu bedurfte es allerdings seit 1999 vieler Anstrengungen, um den demografischen Wandel als ein komplexes Thema zu verstehen. 


\section{Demografischer Wandel als komplexes Thema}

Die Arbeitsgestaltung der 90er-Jahre des letzten Jahrhunderts waren geprägt vom ISO 9000 Boom und von Lean Production, aber auch vom Paradigmenwechsel im Arbeitsschutz hin zu einer salutogenen, auch Ressourcen betrachtenden Sichtweise des Gesundheitserhalts bei der Arbeit. Die Herausforderung der Gestaltung des demografischen Wandels hat sich erst zögerlich in den Köpfen der Praktiker*innen durchgesetzt. Wen interessierte es 1999 schon, wenn der Altersdurchschnitt der Belegschaft im Jahre 2019 etwa bei 50 Jahren liegen wird? Die Feststellung, dass an dieser Entwicklung kaum etwas zu ändern sein wird, ist von der betrieblichen Praxis kaum wahrgenommen worden, zu unterschiedlich waren die Zeithorizonte gesellschaftlicher und betrieblicher Perspektiven.

Die Gestaltung des demografischen Wandels in Unternehmen und Verwaltungen ist eine komplexe, mehrdimensionale Aufgabe, die selbst einen langen Zeithorizont verlangt. Einen weiteren Blick in die Zukunft über einen Fünfjahreshorizont hinaus zu wagen, gehörte damals nicht zum Verhaltensrepertoire vieler Betriebe. Die Arbeitswissenschaft hatte viel zu tun, die komplexe Thematik in die Praxis zu tragen - vergleichbar mit der aktuellen Herausforderung, die Betriebe auf die zunehmende Digitalisierung vorzubereiten. Dabei kennzeichnen die betriebliche Gestaltung von demografischem Wandel und Digitalisierung durchaus gemeinsame charakteristische Merkmale. Diese bestehen darin, dass

- die Kenntnis der Vergangenheit und damit verbundener Diagnosen und Entscheidungen nur begrenzt handlungsinstruktiv sind,

- zu deren Bewältigung eine Vielzahl von häufig unterschiedlichen Maßnahmen notwendig sind, die sämtliche Funktionsbereiche und Funktionsgruppen im Unternehmen betreffen,

- mit langen Lern- und Implementierungszeiten bei häufig schwieriger Koordination umgegangen werden muss, und

- mit zeitweise ungünstigen Relationen von zusätzlichen Erträgen und zusätzlichen Kosten zu rechnen ist. Der Ressourcenaufwand ist beträchtlich und will top down entschieden und bottom up umgesetzt werden.

\subsection{Demografischer Wandel im Diffusionsverlauf von 2000 bis 2030}

Inwiefern der demografische Wandel von der Gesamtheit der Unternehmen erkannt, aufgenommen und umgesetzt worden ist, beschreibt den 
Ausbreitungsprozess über die Zeit (Diffusionsverlauf). Die Geburtenzahl nimmt seit den 50er-Jahren stetig zu, um dann ab 1955 bis etwa 1965 stark anzusteigen und danach wieder bis 1970 stark abzufallen und in ein Niedrigniveau einzupendeln. Etwa ab dem Jahr 2010 hat dieser Verlauf der Geburtenzahlen zu einem stetigen Anstieg der über 50-Jährigen in den Unternehmen geführt, der bis 2020 extrem angestiegen ist, danach etwas flacher ansteigen und wieder absteigen wird. Diese Entwicklung, die aufgrund der vorhandenen Geburten gar nicht zu ändern war und noch ist, lag als Erkenntnis im Jahr 1999 vor. Modis (1998) charakterisiert den Diffusionsverlauf solcher komplexen Themen mit einer S-Kurve, welche sich in drei Phasen unterteilen lässt.

Es beginnt mit einer langen Anlaufphase, d.h. einem gering wachsenden Ausbreitungsgrad. Dem folgt eine Phase beschleunigter Ausbreitung, d.h. es werden am Markt alle Branchen und Betriebsgrößen sowie die unterschiedlichsten Unternehmenstypen erfasst. Diese Beschleunigungsphase geht dann in die Sättigungsphase über, in der die gesamten Akteure der Wirtschaft im Hinblick auf den demografischen Wandel aufgestellt sind, und dies zur Mindestbedingung für die Marktteilnahme geworden ist. Die S-Kurve, welche in Abbildung 1 dargestellt ist und den Diffusionsverlauf des demografischen Wandels in Deutschland wie auch in Westeuropa skizziert, zeigt, dass sich die Wachstumsphase etwa von 2000 bis 2010 erstreckte, um dann eine beschleunigte Phase der Ausbreitung bis etwa 2020 einzuleiten, die dann sukzessive in eine Sättigungsphase übergehen wird.

Abbildung 1: S-kurviger Diffusionsverlauf des demografischen Wandels (Langhoff, 2009)

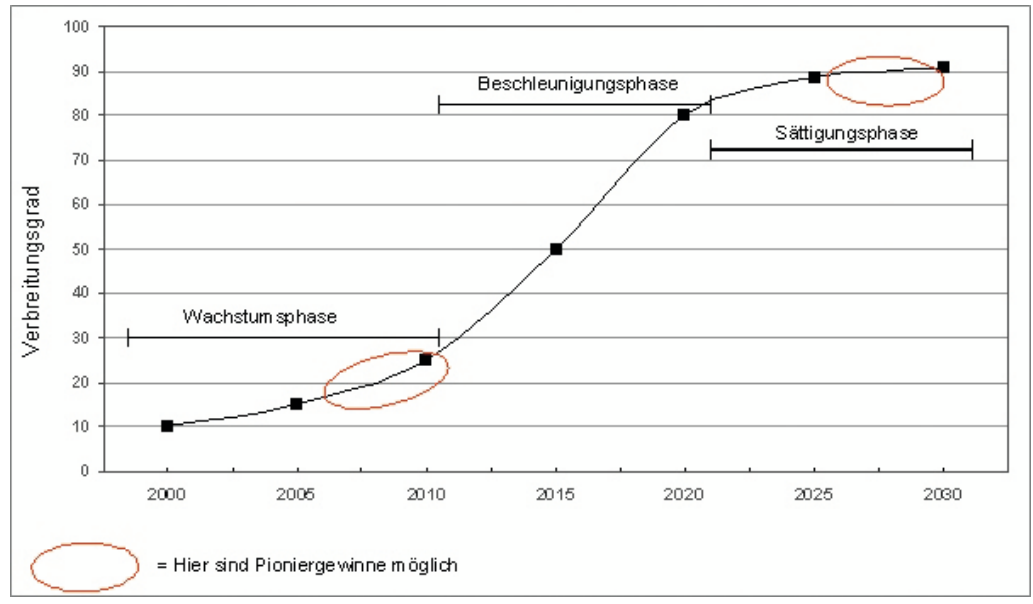


In diesem Diffusionsverlauf gibt es zwei Zeitfenster, in denen Pioniergewinne möglich sind: (1.) im letzten Drittel der Wachstumsphase (2000-2010), wenn die Erfolgsfaktoren schneller und besser (d.h. systematisch) in einem Unternehmen ineinandergreifen und (2.) auf dem Höhepunkt der Sättigungsphase (2020-2030), wenn ein Innovationssprung gelingt, d.h. prinzipiell der Start einer neuen S-Kurve auf höherem Niveau (heute die Kopplung von Digitalisierung bzw. KI bei kommenden Altersstrukturen) erfolgt.

Die Beschleunigungsphase (also 2010-2020) war durch einen harten Mainstream-Wettbewerb um Arbeitskräfte gekennzeichnet. Das hat dazu geführt, dass wir heute in vielen Branchen einen Fach- und Arbeitskräftemangel haben, sowie die psychischen Belastungen der verbliebenen Belegschaften durch zunehmende Arbeitsverdichtung gestiegen sind, und auch das Bildungsniveau der Belegschaften (weniger die formalen Qualifikationen) gesunken ist.

Wie in Abbildung 2 dargestellt, entstehen für den demografischen Wandel somit zwei zeitversetzte zentrale Herausforderungen, d.h. hinterherlaufende S-Kurven: die (1.) Alterung und (2.) Rekrutierung. Beide werden durch die Wanderung der Kohorten, insbesondere der Babyboomer verursacht. Die S-Kurve der Alterung zeigt, dass etwa um das Jahr 2010 eine Beschleunigungsphase im Umgang mit alternden Belegschaften begonnen hat. Dabei stand der Erhalt der Arbeitsfähigkeit der Belegschaften bis zum inzwischen verlängerten Renteneintrittsalter im Vordergrund. Die S-Kurve der Rekrutierung zeigt hingegen, dass hier eine Beschleunigung erst etwa um das Jahr 2015 eintrat und bis 2025 auf einem akut hohen Level zu bleiben scheint.

Gegenwärtig scheiden die Babyboomer jährlich in nie zuvor dagewesener großer Zahl aus den Unternehmen aus. Daran hat auch die Erhöhung des Renteneintritts auf 67 Jahre nichts geändert. Zugleich droht den Unternehmen ein umfassender Abfluss von Know-how und Erfahrung. Darüber hinaus ist es zeitparallel aufgrund des schrumpfenden Erwerbspersonenpotenzials zur Verknappung auf dem Arbeitsmarkt gekommen. Unternehmen bemühen sich seit etwa 2015 ihre Arbeitskräfte verstärkt mit Erwerbsgruppen zu besetzen, die vor allem in der Produktionswirtschaft vorher weniger im Blickpunkt der Rekrutierung standen: Frauen und Ältere. 
Abbildung 2: Differenzierung des Diffusionsverlaufs in S-Kurven für die zentralen Prozesse

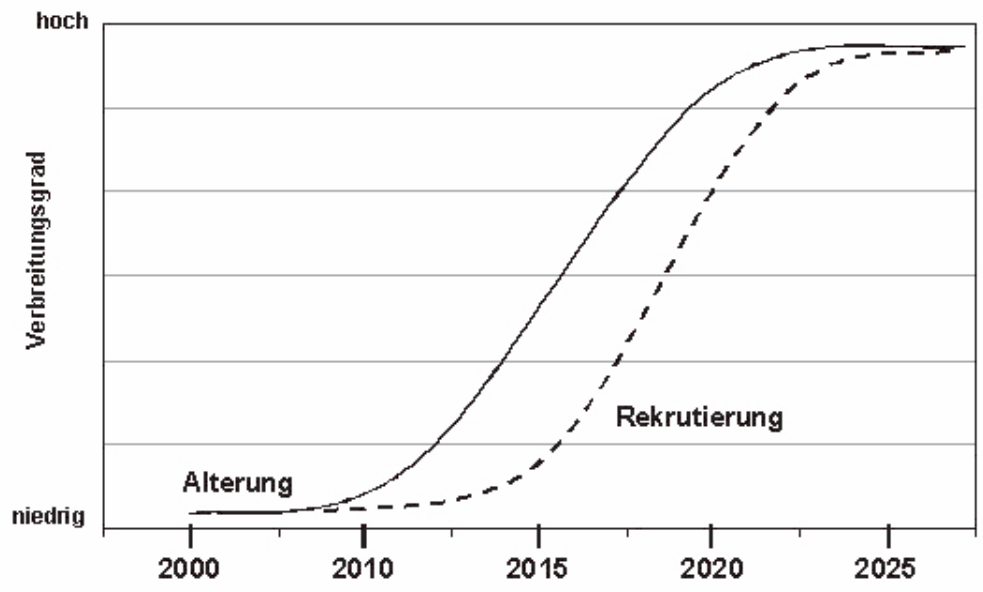

Alterung und Personalbedarf (bzw. -verlust) (Langhoff, 2009)

\subsection{Demografischer Wandel als Wettbewerbsfaktor}

Wie die Konsequenzen, die sich aus der Kohortenwanderung ergeben haben, zeigen, ist die Arbeitsgestaltung im demografischen Wandel längst zu einem Wettbewerbsfaktor geworden. Diejenigen Pionierunternehmen, die sich 2000 bis 2010 als Vorreiterunternehmen dargestellt haben, d.h. eine Managementkompetenz zur Beherrschung des demografischen Wandels gezeigt haben, konnten eine Reihe Erfolgsfaktoren aufweisen, die in dieser Form jetzt kaum mehr möglich sind:

- Solide Analyse und Beurteilung altersbezogener Daten im Unternehmen und dynamischer Unternehmen-Umfeld-Entwicklungen (Verständnis von Alters- und Kohortenpräferenzen, siehe hierzu Konzepte der Altersstrukturanalyse, Langhoff, 2005),

- erfolgreiche Wissensdiffusion (vor allem auch Integration von versäultem technischem, wirtschaftlichem und wissenschaftlichem Wissen),

- konsequente Umsetzung der Prinzipien Individualisierung und Partizipation, 
- Herausbildung einer „Ensemblekompetenz“ quer über alle Funktionsbereiche durch organisationales Lernen (Volkholz \& Langhoff 2008),

- Überführung von organisatorischen Lösungen in Leistungen der Unternehmenskultur - also die Variante mit den höchsten Effizienzgewinnen (Wertschöpfung durch Wertschätzung, siehe hierzu Employer Branding Konzepte) (Brand et al., 2015),

- gezielte Suche nach und Entwicklung von Innovationen (mit zunehmend älteren Innovationsträgern, siehe hierzu auch Konzepte des Usability Engineering bzw. der User Experience (Langhoff et al., 2015)).

1.3 Meilensteine der Diffusion wissenschaftlicher Erkenntnisse in die betriebliche Praxis

Abbildung 3: Drei Bausteine zur Gestaltung des demografischen Wandels: Altersstrukturanalyse, Qualifikationsbedarfsanalyse und Gefährdungs- und Belastungsbeurteilung (Szymanski \& Lange, 2013; INQA 2020)

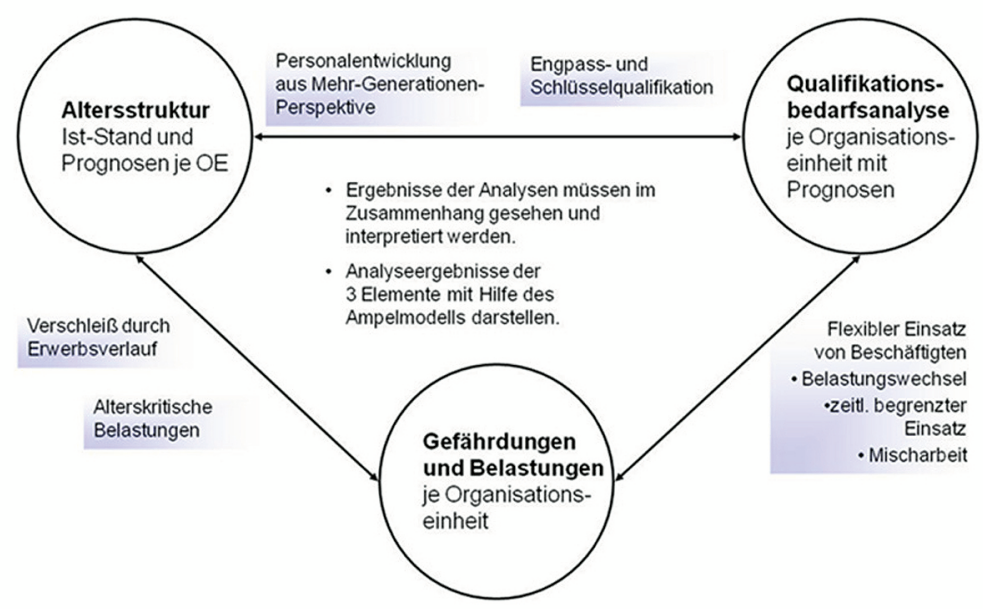

Meilensteine der Diffusion wissenschaftlicher Erkenntnisse in die betriebliche Praxis sind die sogenannten Demografieverträge, die häufig eine andere Bezeichnung tragen (z.B. Tarifvertag zur Förderung altersgerechter Arbeit o.Ä.), (siehe hierzu auch den Beitrag von Guggemos \& Conrads in diesem Band). Ziel der im Zeitraum von etwa 2005 bis 2015 abgeschlossenen Demografieverträge war es, die Arbeitsfähigkeit der Belegschaften bis 
zum Regelrenteneintritt aufrechtzuerhalten. Dazu wurden Handlungsfelder und wissenschaftlich validierte Instrumente einer systematischen Personalarbeit für alternde Belegschaften beschrieben (Altersstrukturanalyse, Gefährdungs- und Belastungsanalyse, Qualifizierungsbedarfsanalyse). Damit wurde dem demografischen Wandel das Bedrohungspotenzial genommen und Chancen für eine arbeitswissenschaftliche fundierte alters- und alternsgerechte Arbeitsgestaltung sowie eine nachhaltige Personalpolitik eröffnet (siehe Abbildung 3).

Die Initiative Neue Qualität der Arbeit (INQA) (2014) hat einen Überblick über Demografieverträge publiziert. Danach haben zu diesem Zeitpunkt vor allem Tarifvertragsparteien aus der Industrie Demografieverträge abgeschlossen. Dieser Überblick sollte aktualisiert werden. Tarifpolitische Initiativen sind, die arbeitswissenschaftliche Erkenntnisse in der Praxis zur Anwendung bringen. Damit haben die Tarif- und Sozialpartner*innen eine wichtige Promotorenrolle übernommen, die insgesamt sowohl von den Arbeitgeber*innen wie auch von den Arbeitnehmer"innen auf betrieblicher Ebene positiv aufgenommen worden ist.

Abbildung 4: Regelungen zu Handlungsfeldern in Tarifverträgen (INQA, 2014)

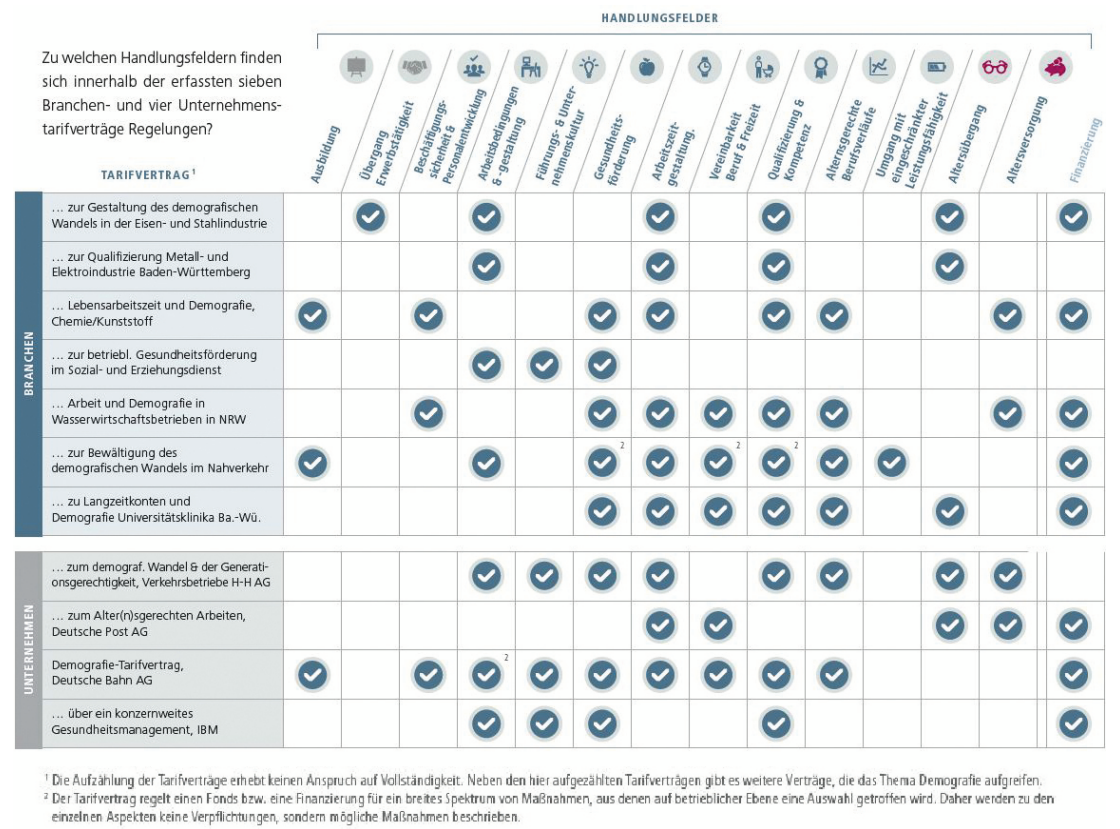


Wie in Abbildung 4 zu sehen ist, sind die Handlungsfelder Arbeitszeitgestaltung, Gesundheitsförderung und Qualifizierung in neun von elf Tarifverträgen behandelt worden. Arbeitsgestaltung selbst war Bestandteil von acht Tarifverträgen. Arbeitszeitgestaltung ist selbstverständlich ein Teil der Arbeitsgestaltung, ist aber aufgrund der Prioritätensetzung von den Arbeitgeber*innen als eigenständiges Handlungsfeld aufgenommen worden (INQA/BAuA, 2014, S. 22).

Ein weiteres wichtiges Instrument für die betriebliche Gestaltung des demografischen Wandels, welches gegen Ende der 90er-Jahre des letzten Jahrhunderts entwickelt worden ist, ist die Altersstrukturanalyse (Langhoff 2005), wobei damit nicht allein die Darstellung der Altersstruktur der Belegschaft gemeint ist.

\section{Abbildung 5: Altersstrukturanalyse: Bestandsaufnahmebogen, Schlüsselvaria- blen und Hinweise für Gestaltungsfelder im Überblick (Langhoff, 2009)}

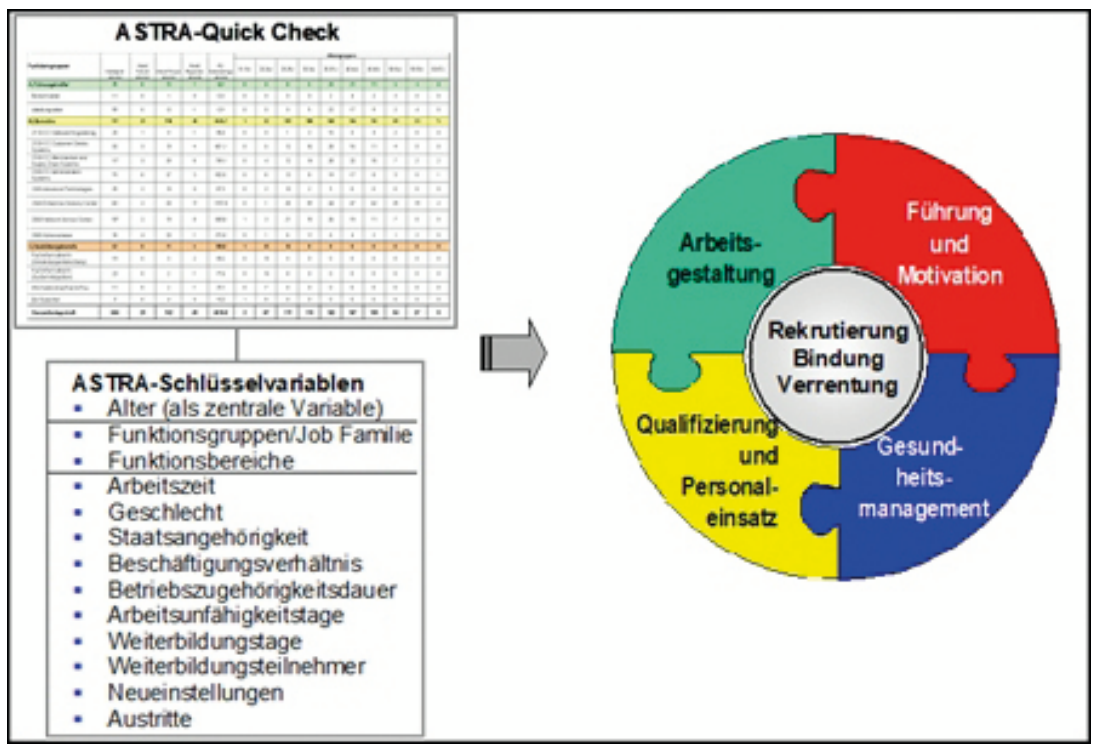

Im Grunde muss man sich jede betriebliche Variable nach Alter anschauen: Jobfamilien, Funktionsbereiche, Arbeitszeit, Geschlecht, Beschäftigungsverhältnis, Betriebszugehörigkeitsdauer, AU-Tage, BEM-Fälle, Weiterbildungstage, Weiterbildungsteilnehmer*innen, Neueinstellungen und Austritte (siehe Abbildung 5). Die Altersstrukturanalyse wurde schnell 
zum verpflichtenden Basisinstrument in den o.g. Demografieverträgen (sowie auch in der DGUV Vorschrift 2). Ihren Analyseergebnissen wurden Gestaltungsmaßnahmen zugeordnet und auf die Handlungsfelder Arbeitsgestaltung, Gesundheit, Qualifizierung sowie Führung verteilt (Langhoff, 2009).

Es gilt, mit der demografischen Brille auf das betriebliche Geschehen zu blicken, den Ist-Zustand zu erfassen, Auffälligkeiten zu identifizieren und Prognoseverläufe abzuleiten. Mit den Ergebnissen der Altersstrukturanalyse wurde den meisten Betrieben schockartig klar, was auf sie in den nächsten fünf bis zehn Jahren zukommt, der Erhalt der Arbeitsfähigkeit der älteren Beschäftigten, und mit der zunehmenden Verrentung der Älteren auch beträchtliche Rekrutierungsanforderungen (siehe Abbildung 6).

Abbildung 6: Zunahme des Anteils über 50-Jähriger sowie verrentungsbezogener Rekrutierungsbedarf bei einer Rente ab 65 Jahren: anonymisiertes Beispiel eines mittelständischen Produktionsbetriebes aus dem Jahr 2002 (Langhoff, 2009)

\begin{tabular}{|c|c|c|c|c|c|c|c|}
\hline \multirow[b]{2}{*}{ Funktions qruppen } & \multicolumn{2}{|c|}{$\begin{array}{c}\text { Ersatzbedarf } \\
\text { (Rente ab65 J.) }\end{array}$} & \multicolumn{5}{|c|}{ Anteil über 50-Jährige } \\
\hline & bis 2007 & $2008-2012$ & 2002 & 2007 & $\begin{array}{c}\text { Delt a } \\
2002-07\end{array}$ & 2012 & $\begin{array}{c}\text { Delta } \\
2002-12\end{array}$ \\
\hline \multirow{2}{*}{ Führungskräfte } & $14,3 \%$ & $8.3 \%$ & $35.7 \%$ & $58,3 \%$ & \multirow[t]{2}{*}{$22,6 \%$} & $90.9 \%$ & \multirow[t]{2}{*}{$55,2 \%$} \\
\hline & 2 & 1 & 5 & 7 & & 10 & \\
\hline \multirow{2}{*}{$\begin{array}{l}\text { Technische Angestellte } \\
\text { fohne Meister. Vorameiter }\end{array}$} & $20,0 \%$ & $25,0 \%$ & $60,0 \%$ & $50,0 \%$ & \multirow[t]{2}{*}{$-10,0 \%$} & $66,7 \%$ & \multirow[t]{2}{*}{$6,7 \%$} \\
\hline & 1 & 1 & 3 & 2 & & 2 & \\
\hline \multirow{2}{*}{$\begin{array}{l}\text { Kaufmännische } \\
\text { Anqestellte }\end{array}$} & $0,0 \%$ & $0,0 \%$ & $0,0 \%$ & $0,0 \%$ & \multirow[t]{2}{*}{$0,0 \%$} & $100,0 \%$ & \multirow[t]{2}{*}{$100,0 \%$} \\
\hline & 0 & 0 & 0 & 0 & & 1 & \\
\hline \multirow{2}{*}{ Angeste lte Me ister } & $33,3 \%$ & $0,0 \%$ & $33,3 \%$ & $50,0 \%$ & \multirow[t]{2}{*}{$16,7 \%$} & $100,0 \%$ & \multirow[t]{2}{*}{$66,7 \%$} \\
\hline & 1 & 0 & 1 & 1 & & 2 & \\
\hline \multirow{2}{*}{ Vorarbeiter u.ä. } & $0,0 \%$ & $0,0 \%$ & $20,0 \%$ & $80,0 \%$ & \multirow[t]{2}{*}{$60,0 \%$} & $100,0 \%$ & \multirow[t]{2}{*}{$80,0 \%$} \\
\hline & 0 & 0 & 1 & 4 & & 5 & \\
\hline \multirow{2}{*}{ Mitarbeiter } & $7.4 \%$ & $15,3 \%$ & $38.5 \%$ & $49,2 \%$ & \multirow[t]{2}{*}{$10,6 \%$} & $50.5 \%$ & \multirow[t]{2}{*}{$12,0 \%$} \\
\hline & 9 & 18 & 47 & 58 & & 53 & \\
\hline \multirow{2}{*}{ Kaufmännische Angestelle } & $18,2 \%$ & $44.4 \%$ & $90,9 \%$ & $88,9 \%$ & \multirow[t]{2}{*}{$-2,0 \%$} & $80,0 \%$ & \multirow[t]{2}{*}{$-10,9 \%$} \\
\hline & 2 & 4 & 10 & 8 & & 4 & \\
\hline \multirow{2}{*}{$\begin{array}{l}\text { Facharbeiter (fachspezif., } \\
\text { d.h. ohne Bäcker.Friseur u.ä.) }\end{array}$} & $14,8 \%$ & $10,7 \%$ & $44,4 \%$ & $35,7 \%$ & \multirow[t]{2}{*}{$8,7 \%$} & $300 \%$ & \multirow[t]{2}{*}{$-14,4 \%$} \\
\hline & 4 & 3 & 12 & 10 & & 9 & \\
\hline \multirow{2}{*}{ Ar und Ung elernte } & $36 \%$ & $136 \%$ & $298 \%$ & $49,4 \%$ & \multirow[t]{2}{*}{$19,6 \%$} & $57,1 \%$ & \multirow[t]{2}{*}{$27,4 \%$} \\
\hline & 3 & 11 & 25 & 40 & & 40 & \\
\hline \multirow{2}{*}{ Ges amtbelegschaft } & $7,8 \%$ & $14,1 \%$ & $36,9 \%$ & $48,1 \%$ & \multirow[t]{2}{*}{$11,3 \%$} & $52,1 \%$ & \multirow[t]{2}{*}{$15,2 \%$} \\
\hline & 11 & 19 & 52 & 65 & & 63 & \\
\hline
\end{tabular}

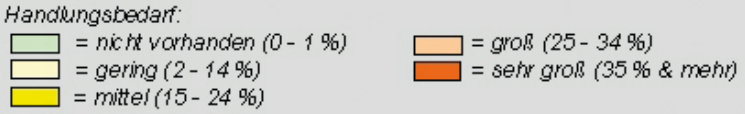

Abbildung 6 zeigt die beiden eingangs erwähnten Herausforderungen Alterung und Rekrutierung, die sich zeitlich versetzt für das Unternehmen 
darstellen. Im Zeitraum 2002 bis 2012 steigt der Anteil der über 50-Jährigen stark an, während sich im gleichen Zeitraum der Ersatzbedarf (noch) in Grenzen hält. Nach 2012 werden die Älteren in großem Umfang verrentet, während gleichzeitig der Ersatzbedarf so stark ansteigt, dass er kaum zu decken ist.

Das Beispiel des mittelständischen Produktionsbetriebes ist insofern typisch für die Zeit, weil zwischen 1999 und 2009 der Erhalt der Arbeitsfähigkeit der alternden Belegschaften im Zentrum stand. Das wichtigste Instrumentarium hierfür war und ist nach wie vor die Gefährdungsbeurteilung nach $₫ 5$ ArbSchG.

Körperliche Belastungen wurden nur selten erhoben und bewertet. Die Leitmerkmalmethode, heute ein Standard zur Bewertung körperlicher Belastungen, wurde zu Beginn der 2000er-Jahre von ersten Pionierunternehmen eingesetzt. Psychische sowie alternskritische Belastungen fanden noch seltener Berücksichtigung. Szymanski und Lange (2014) waren die ersten, die die Gefährdungsbeurteilung mit der demografischen Brille betrachtet, alterskritische Gefährdungen herausgearbeitet, und bei der Maßnahmenplanung alternsbedingte, negative Veränderungen, wie:

- die Abnahme von Muskelkraft und Bewegungs- und Reaktionsgeschwindigkeit,

- die Minderung des Seh- und Hörvermögens,

- das Absinken der Dauer- und Höchstleistungsfähigkeit berücksichtigt haben (siehe Abbildung 7).

In diesem Beitrag stehen die psychischen Belastungen aus Platzgründen im Hintergrund (dazu u.a. Breutmann 2018). Die hohe Bedeutung dieser Thematik, auch vor dem Hintergrund des demografischen Wandels, hat dazu geführt, dass von Gesetzgeberseite psychische Belastungen 2013 in das Arbeitsschutzgesetz explizit aufgenommen wurden. Auch heute, im Jahr 2020 gibt es hierzu außerordentlichen Handlungsbedarf (vgl. Beck/Schuller 2020), wobei die Dienstleistungswirtschaft der Produktionswirtschaft hinterherhinkt. Wird bei der Ermittlung psychischer Belastungen eine schriftliche Befragung durchgeführt, so sind die Ergebnisse zwingend nach Altersgruppen auszuwerten, da sich i.d.R. signifikante Unterschiede ergeben, die sich dann auf die zielgruppenbezogene Maßnahmenplanung auswirken. 
Abbildung 7: Bewertungsraster alternskritischer Gefährdungen und Belastungen (Szymanski und Lange, 2013)

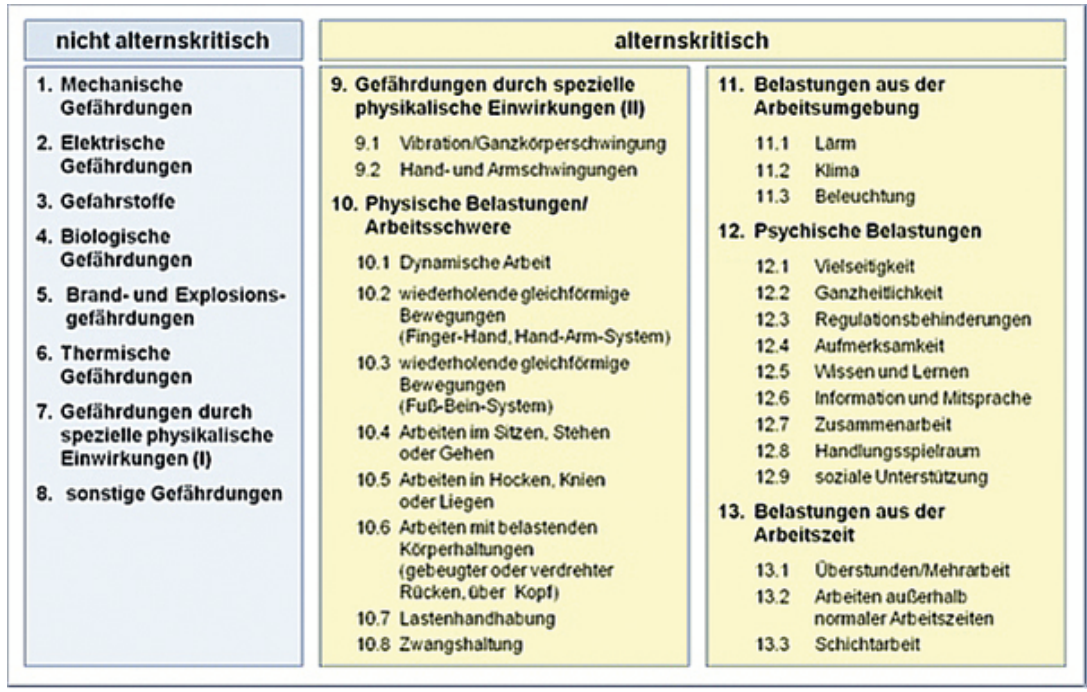

Neben der Altersstrukturanalyse und der Gefährdungsanalyse ist als drittes wichtiges Instrument die Qualifikationsbedarfsanalyse zu nennen. Angemessener wäre eigentlich die Bezeichnung Qualifizierungsbedarfsanalyse, weil es vielmehr um die Passung von Anforderungen und Kompetenzen sowie um Kompetenzentwicklung geht, und nicht um den Erwerb eines Qualifikationszertifikats. Die demografiesensible Personalarbeit hat in diesem Feld in den 20 Jahren zwischen 2000 und 2020 wichtige Erfahrungen gemacht. Die Unternehmen haben insbesondere erkennen müssen, dass ein schleichender Prozess wie die Alterung der Belegschaft unvermittelt eine enorme Tragweite entwickeln kann, z.B. wenn es nicht mehr gelingt, auf dem Arbeitsmarkt den Rekrutierungsbedarf zu decken. Dadurch ist in vielen betroffenen Betrieben die Aufmerksamkeit für Qualifizierungsnotwendigkeiten und -chancen sowie generell die Kompetenzentwicklung der eigenen Belegschaft gestiegen. Mit Vorreiterunternehmen konnten Strategien zur gezielten Förderung interner Qualifizierung entwickelt werden (INQA 2016). 
Folgende Leitfragen zur Qualifizierung alternder Belegschaften haben sich bewährt:

- Wie können die vorhandenen Kompetenzen erfasst werden (fachlich, sozial, methodisch, insbesondere Erfahrungswissen)?

- Erhalten alle Mitarbeiter*innen, unabhängig vom Alter, die gleiche Chance sich zu qualifizieren und ihre Kompetenzen zu erweitern?

- Wie können (gering qualifizierte) Ältere mit positiven Verstärkern (Gefühlen) zum Lernen angeregt werden?

- Wird gezielt der Wissenstransfer zwischen älteren, erfahrenen Mitarbeiter*innen und dem Nachwuchs gefördert?

- Wie kann neues Wissen im Unternehmen verbreitet werden (Kulturwandel in Richtung lernende Organisation)?

- Welche Qualifikationen werden in Zukunft gefragt und welche Anforderungen an die Weiterbildung (älterer) Beschäftigter ergeben sich daraus (Weiterbildungsbereitschaft Älterer - Weiterbildungsangebote für Ältere)?

- Wie lange üben die Mitarbeiter*innen ihre jetzige Tätigkeit aus?

- Wie viele Tätigkeitswechsel haben Mitarbeiter“innen durchlaufen?

- Wie können Ältere gezielt als Innovationsträger genutzt werden?

Um den o.g. Fragestellungen näherzukommen, wurde das bereits bekannte Prinzip der demografischen Brille auf eine konventionelle Personaleinsatzmatrix (oder auch Qualifikationsmatrix) angewendet, d.h. die zentrale Variable ist das Alter der Beschäftigten. Dieses wird in Beziehung gesetzt zum Aufgabeneinsatz, zum Belastungsgehalt und zum Qualifikationsstand der Beschäftigten. Das Instrument ist gut mit herkömmlichen Personaleinsatzkonzepten in Unternehmen mittlerer Größe kombinierbar und dient dem Umdenken und der Neugestaltung des Personaleinsatzes. Der Blick wird auf alterskritische Tätigkeiten gelenkt. Bei identifizierten Belastungshäufungen kann über Schulungsmaßnahmen bzw. neue Arbeitseinsatzstrategien nachgedacht und entschieden werden. 
Abbildung 8: Personaleinsatzmatrix für den alternsgerechten Personaleinsatz (Reindl et al. 2004)

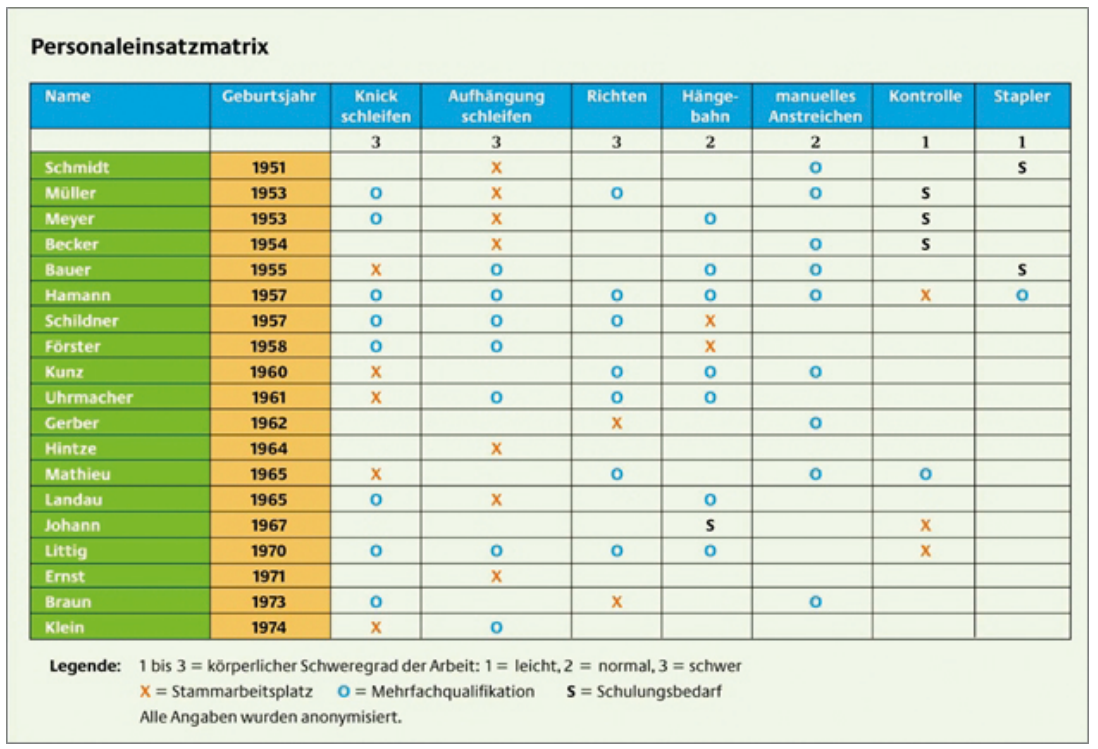

Die Matrix macht auf den ersten Blick deutlich, was passiert wenn ältere Beschäftigte besonders belastende Tätigkeiten ausüben. Dies fällt in herkömmlichen Personaleinsatzkonzepten sonst nicht auf. Außerdem können Qualifizierungsbedarfe leicht erkannt werden, die zu einer Entlastung bzw. zu einem neuen Aufgabenzuschnitt mit wechselnden Belastungen führen können. Abbildung 8 zeigt einen Arbeitsbereich mit den dort tätigen Mitarbeitenden nach Alter gruppiert. Des Weiteren sind die in dem Arbeitsbereich vorkommenden Arbeitsaufgaben eingetragen und nach körperlichem Schweregrad bewertet. Es wird der jeweilige Personaleinsatz abgebildet in Form des Stammarbeitsplatzes, der weiteren „vorgehaltenen“ Qualifikation und des Schulungsbedarfs. In dem o.g. Betriebsbeispiel wird z.B. deutlich, dass die ältesten Mitarbeiter*innen allesamt die körperlich schwere Arbeit „Aufhängung schleifen“ ausüben, während ihnen auf der anderen Seite körperlich leichte Tätigkeiten zum Ausgleich fehlen. Hier besteht also akuter Handlungsbedarf. Oftmals erscheinen personenbezogene Aufgabenzuschnitte geradezu historisch gewachsen. Es gilt, dies im Rahmen einer Prüfung auf Demografietauglichkeit festzustellen und durch geeignete Schulungsmaßnahmen und Neubestimmungen von belastungsgerechten Aufgabenzuschnitten zu korrigieren. Dabei stellt die Kom- 
munikation im Betrieb und die Überzeugungskraft für eine gesunderhaltende Arbeit eine große Herausforderung dar. Ein typisches Beispiel zeigt die Schwierigkeiten bei der Wandlung von stark belastenden hin zu gesundheitsstabilen Schichtmodellen (siehe hierzu auch Langhoff \& Satzer, 2017).

Die Personaleinsatzmatrix für den alternsgerechten Personaleinsatz ist sehr einfach aufgebaut und in verschiedensten Branchen eingesetzt worden. Eine Erweiterung auf mentale Belastungen (leicht, mittel, schwer) wurde später vorgenommen (siehe auch Reindl. u.a. 2004 sowie Bertelsmann Stiftung/BDA, 2005).

Altersstrukturanalyse, Gefährdungsanalyse und Qualifizierungsbedarfsanalyse stellen die Bausteine der Triade dar, die auch in den ersten Demografieverträgen der Eisen- und Stahlindustrie und der Metallindustrie gefordert wurden. Ihre parallele, gemeinsame Bearbeitung ist höchst effizient und ermöglicht Maßnahmen einer ganzheitlichen alternsgerechten Arbeitsgestaltung.

\section{Vom Defizit-zum Kompetenzmodell}

Etwa noch 2010 wurde die zunehmende Alterung im Betrieb vor allem als Risiko gesehen. Schrittweise hat sich seither ein Umdenken eingestellt, der nicht mehr von einer verallgemeinernden Defizithypothese des Alters ausgeht. Die Altersentwicklung wird als kontinuierlicher, lebenslanger Prozess verstanden, bei dem die biologischen Altersphasen nicht eindeutig durch gewisse Zuwächse und Verluste definiert werden können. Vielmehr werden sowohl altersabhängige als auch altersunabhängige Faktoren der Entwicklung betrachtet, die in verschiedenen Lebensbereichen und Verhaltensebenen unterschiedlich ausgeprägt sein können (Baltes, Mittelstraß \& Staudinger, 1994). Hierbei spielen nicht nur biologische Faktoren eine Rolle, sondern es wird zwischen intra- und interindividuellen Unterschieden hinsichtlich der Lebensbedingungen und Erfahrungen, sowie zwischen sozialen und kulturellen Faktoren differenziert (Baltes, 1990). Dieser differenzierte Blick macht deutlich, dass die gesamte Lebensspanne von einem Wechselspiel zwischen Auf- und Abbauprozessen begleitet wird und sich das Individuum durch die Anpassungs- und Änderungsfähigkeit an altersbedingte Beeinträchtigungen, veränderte Situationen und neue Voraussetzungen anpassen kann. Auf Basis dieser Betrachtungen ist das Kompetenzmodell (des Alterns) formuliert worden (siehe Abbildung 9). 
Abbildung 9: Gegenüberstellung des früheren Ansatzes des Defizitmodells vom Altern mit dem heute gültigen Kompetenzmodell des Alterns (Langhoff et al., 2015)

\begin{tabular}{|l|l|}
\hline Defizitmodell des Alterns & Kompetenzmodell des Alterns \\
\hline Bis 70er-Jahre des 20. Jahrhunderts & $\begin{array}{l}\text { Von 90er-Jahre des 20. Jahrhun- } \\
\text { derts bis heute }\end{array}$ \\
\hline Altern als defizitärer Verlauf & $\begin{array}{l}\text { Altern als ein Abschnitt des lebens- } \\
\text { langen Entwicklungsprozesses }\end{array}$ \\
\hline $\begin{array}{l}\text { Gekennzeichnet durch Verluste, } \\
\text { Mängel und Defizite }\end{array}$ & $\begin{array}{l}\text { Gekennzeichnet durch ein Wech- } \\
\text { selspiel von Gewinnen und Verlus- } \\
\text { ten }\end{array}$ \\
\hline Biologische Faktoren & $\begin{array}{l}\text { Multifaktoriell (biologische, intra- } \\
\text { \& interindividuelle, soziale und } \\
\text { kulturelle Faktoren) }\end{array}$ \\
\hline Verallgemeinerung der Ergebnisse & Differenzierung der Ergebnisse \\
\hline
\end{tabular}

Zwar schließt das Kompetenzmodell altersbedingte und altersspezifische Defizite nicht aus, allerdings wird hierbei zwischen verschiedenen Bereichen (z.B. kognitive Fähigkeiten, soziale Kompetenzen etc.) unterschieden. So können zwar Verluste z.B. im Bereich der Verarbeitungsgeschwindigkeit oder der Kapazität des Arbeitsgedächtnisses gemessen werden, jedoch sind es gerade ältere Menschen, die hinsichtlich einer Vielzahl von Schlüsselkompetenzen wie z.B. kommunikative und soziale Fähigkeiten ein hohes Potenzial aufweisen (Prezewowsky, 2007). Hinzu kommt die Erkenntnis, dass die Lernfähigkeit nicht schlechter als bei jüngeren Menschen ist. Das Kompetenzmodell des Alterns ist aus wissenschaftlicher Sicht gültig und stellt daher die Basis für die Betrachtung betrieblicher Alterungsprozesse im Rahmen der alternsgerechten Arbeitsgestaltung dar.

Um die wichtigsten Erkenntnisse der Arbeitsgestaltung zusammenzufassen, empfiehlt sich die Struktur und die Elemente des Arbeitssystemmodells zu wählen, wie es auch von der Gemeinsamen Deutschen Arbeitsschutzstrategie (2017) als Grundlage der Gefährdungsbeurteilung oder auch in der DIN EN ISO 6385 (2004) verwendet wird (siehe Abbildung $10)$. 
Abbildung 10: Merkmalsbereiche der Gefährdungsbeurteilung nach GDA, hergeleitet aus der ISO 6385 (GDA, 2017)

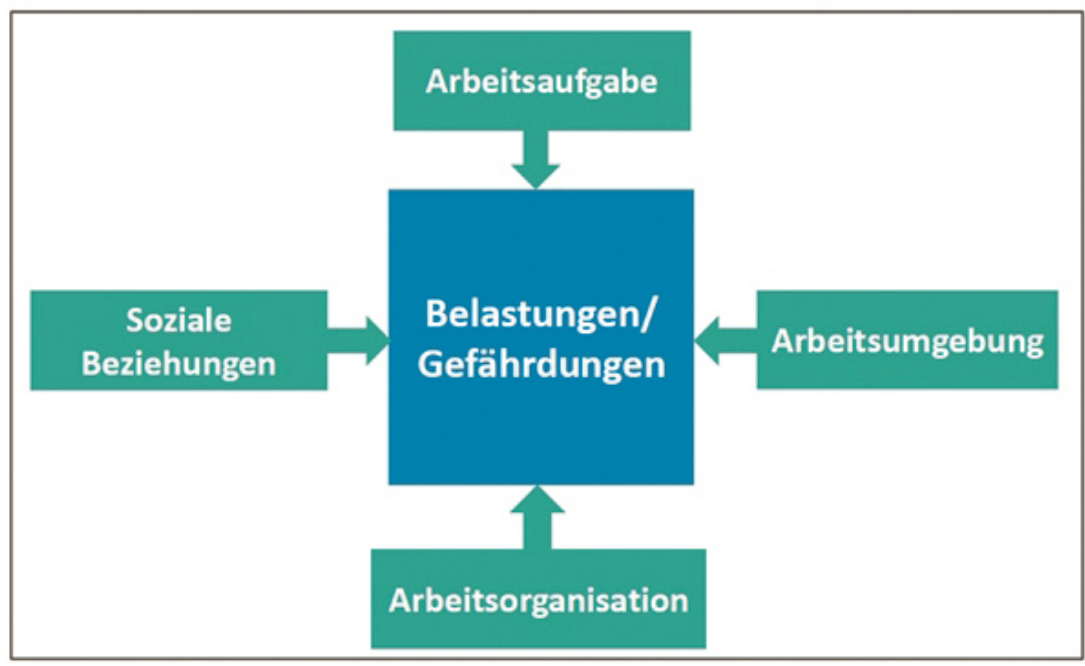

Neben den Ansatzpunkten einer alternsgerechten Arbeitsgestaltung, die langfristig positive Wirkungen für alle Altersgruppen haben, werden im Folgenden Merkmale genannt, deren Bedeutung mit dem Alter zunimmt (BAuA, 2017), also eher auf eine altersgerechte Arbeitsgestaltung für ältere Arbeitnehmer*innen ausgerichtet ist.

\section{Arbeitsaufgabe:}

- Handlungsspielraum/Autonomie ermöglichen (generell und besonders bei schnell zu verarbeitenden Informationen)

- Anforderungsvielfalt ermöglichen

- Aufgaben zuweisen, die Erfahrungswissen erfordern

- Unterforderung vermeiden

- Aufgaben lernförderlich gestalten

\section{Arbeitsorganisation:}

- Ausreichende Erholungszeiten und Gelegenheiten zur Regeneration ermöglichen (z.B. bei schwerer körperlicher Arbeit; nach Nachtschichtblöcken) 
- Schichtarbeit gesundheitsverträglich gestalten und reduzieren

- Gleitende Ausstiege aus dem Erwerbsleben ermöglichen

\section{Arbeitsumgebung:}

- Beleuchtungsstärke und Kontraste erhöhen, Blendung vermeiden, Hintergrundgeräusche und dauerhaften Lärm reduzieren, Eindeutigkeit von Signalen erhöhen

- Ergonomische Arbeitsmittel, Arbeitshilfen und Assistenzsysteme nutzen, Autonomie und Partizipation erhöhen

- Mischarbeit mit organisiertem Belastungswechsel

- Zwangshaltungen, übermäßiges Beugen, Bücken und Verdrehen vermeiden

\section{Soziale Beziehungen:}

- Soziale Unterstützung durch Kolleg*innen und Vorgesetzte fördern

- Weitergabe von Wissen und Erfahrung fördern

- Auf respektvollen und wertschätzenden Umgang achten, Feedback zur Arbeitsleistung geben

Die beschriebenen Gestaltungshinweise dienen dazu, die Arbeitsfähigkeit bis zum Regelrenteneintritt und darüber hinaus zu erhalten. Hierbei sind pauschale Gestaltungslösungen meist wenig erfolgversprechend, da die körperlichen und psychischen Unterschiede zwischen Individuen im Altersgang zunehmen und die Arbeitsfähigkeit mit dem Alter zunehmend differenziert. Dies ist durch verschiedene Tätigkeitsanforderungen über das Arbeitsleben hinweg verursacht, aber auch durch den individuellen Lebenswandel geprägt.

\section{Neue und alte Anforderungen an das nächste Jahrzehnt}

Die arbeitswissenschaftlich fundierte Arbeitsgestaltung ermöglicht es weitgehend, alternde Belegschaften arbeitsfähig zu erhalten. Insofern trägt sie auch dazu bei, die aufkommenden Rekrutierungsanforderungen durch unsere schrumpfende Gesellschaft ein Stück weit abzumildern und maximale Beschäftigungspotenziale auszuschöpfen. Die notwendigen arbeitswissenschaftlichen Erkenntnisse und auch Praxisbeispiele sind hierfür vorhanden bzw. in den letzten 20 Jahren erarbeitet worden. Leider tut sich die betriebliche Praxis mit der Diffusion der Erkenntnisse teilweise immer noch 
schwer, z.B. bei der gesundheitsverträglichen Schichtarbeitsgestaltung (siehe hierzu Langhoff \& Satzer, 2017). Auch die Thematik „Zwangshaltungen, übermäßiges Beugen, Bücken und Verdrehen“ ist nach wie vor auf konstant hohem Niveau (Institut DGB-Index Gute Arbeit, 2019; Gebhardt et al., 2019). Ob die technologischen Entwicklungen in der Mensch-Roboter-Kollaboration oder auch der Exoskelette neuen Fortschritt ermöglichen, wird sich zeigen.

Es ist bedauerlich, dass nur wenige Führungskräfte in den Unternehmen die beobachtbaren Treiber für die Realisierung arbeitswissenschaftlicher Erkenntnisse sind, sondern eher die Betriebsräte, die sich für gesunde Arbeitsbedingungen einsetzen und kämpfen, manchmal sogar gegen die mehrheitliche Belegschaftsmeinung. Keine Personengruppe fördert mehr die breitenwirksame Umsetzung des Arbeitsschutzgesetzes, des Arbeitssicherheitsgesetzes, der Betriebssicherheitsverordnung, der Arbeitsstättenverordnung etc. als die Betriebsräte, die häufig in Einigungsstellen und vor dem Arbeitsgericht gesundheitsstabile Arbeitsbedingungen erzwingen müssen. Ein Umdenken in den Unternehmensführungen ist hinsichtlich der Gestaltung gesunder Arbeit kaum in Sicht, was demnach auch nicht die ,Sisyphusarbeit ${ }^{\star}$ der betrieblichen Arbeitsschutzakteure verändern wird.

Insgesamt wird die Bewältigung des demografischen Wandels, der noch mindestens zehn Jahre präsent sein wird, in die neuen Mega-Trends Globalisierung, Digitalisierung (Industrie 4.0; KI) und Klimawandel überführt werden. Insbesondere die nicht vorhandenen Personalressourcen (aufgrund fehlender Geburten) werden den Unternehmen Schwierigkeiten bereiten. In diesem Zusammenhang sind soziale Innovationen möglich mit Konzepten der Arbeitgeberattraktivität, Konzepten des lebensphasenorientierten Arbeitens und der Gestaltung von Erwerbsverläufen (Tätigkeitswechsel; siehe hierzu Rosetti \& Langhoff, 2015; Rump \& Eilers, 2017; sowie Bundesverband der Berufsförderungswerke, 2019). 


\section{Literatur}

Baltes, P. B., Mittelstraß, J. \& Staudinger, U. M. (1994): Alter und altern. Ein interdisziplinärer Studientext zur Gerontologie. Berlin: de Gruyter.

Baltes, P. B. (1990): Entwicklungspsychologie der Lebensspanne: Theoretische Leitsätze. Psychologische Rundschau, 1, S. 1-24.

Baltes, P. B. \& Baltes, M. M. (1994): Gerontologie: Begriff, Herausforderung und Brennpunkte. In: P. B. Baltes, J. Mittelstraß, U. M. Staudinger (Hrsg.): Zukunft des Alterns und gesellschaftliche Entwicklung. Berlin: de Gruyter, S. 1-34.

Beck, D. \& Schuller, K. (2020): Gefährdungsbeurteilung psychischer Belastung in der betrieblichen Praxis. Erkenntnisse und Schlussfolgerungen aus einem Feldforschungsprojekt. In: baua: Bericht kompakt, 1. Auflage. Dortmund: Bundesanstalt für Arbeitsschutz und Arbeitsmedizin, S. 3.

Breutmann, N. (2018): Bedeutung der psychischen Gefährdungsbeurteilung. In: G. Richter, C. Hecker, C. Hinz (Hrsg.): Für die Initiative Neue Qualität der Arbeit: Produktionsarbeit in Deutschland - mit alternden Belegschaften. Berlin: Bertelsmann Stiftung, S. 208-218.

Bundesvereinigung der Deutschen Arbeitgeberverbände (Hrsg.) (2005): Erfolgreich mit älteren Arbeitnehmern. Strategien und Beispiele für die betriebliche Praxis, 2. Auflage. Gütersloh.

Bundesanstalt für Arbeitsschutz und Arbeitsmedizin (BAuA) (Hrsg.) (2017): Alterns- und altersgerechte Arbeitsgestaltung. Grundlagen und Handlungsfelder für die Praxis. Dortmund.

Bundesverband Deutscher Berufsförderungswerke (Hrsg.) (2019): Erwerbsverläufe präventiv gestalten. Berlin.

DIN EN ISO 6385: (2004): Grundsätze der Ergonomie für die Gestaltung von Arbeitssystemen.

Gebhardt, H. J., Schäfer, A., Serafin, P., Klußmann, A. \& Lang, K.-H. (2019): Zustandsanalyse physischer Belastung in Deutschland. In: MEGAPHYS - Mehrstufige Gefährdungsanalyse physischer Belastungen am Arbeitsplatz (Band 1). Dortmund, S. 81-121.

Gemeinsame Deutsche Arbeitsschutzstrategie (GDA) (2017): Empfehlungen zur Umsetzung der Gefährdungsbeurteilung psychischer Belastung, 3. überarbeitete Auflage. Berlin.

INQA, Initiative Neue Qualität der Arbeit (Hrsg.) (2014): Monitor. Tarifverträge zur Gestaltung der Qualität der Arbeit. Ein aktueller Überblick über Vereinbarungen zu alternsgerechten und demografiefesten Arbeitsbedingungen. Berlin.

INQA, Initiative Neue Qualität der Arbeit (Hrsg.) (2016): Interne Potenziale Kompetenzen von Mitarbeiterinnen und Mitarbeitern erkennen, nutzbar machen, entfalten. Von K. Rosetti und T. Langhoff. https:/www.inqa.de/SharedDocs/do wnloads/webshop/interne-potenziale-ireq.pdf (abgerufen am 17.06.2020). 
INQA, Initiative Neue Qualität der Arbeit (Hrsg.) (2020): Teams und Belegschaften systematisch entwickeln. Drei Werkzeuge für Qualifizierung, gute Arbeitsgestaltung und Nachfolgeplanung im Mittelstand. Von A. Lange, H. Szymanski, G. Kolbe, I. Mühlenbrock. Berlin, https:/www.inqa.de/SharedDocs/downloads/tea ms-und-belegschaften-systematisch-entwickeln.pdf (abgerufen am 17.06.2020).

Institut DGB-Index Gute Arbeit (Hrsg.) (2019): Körperlich harte Arbeit. So beurteilen die Beschäftigten ihre Belastungen. Ergebnisse einer Sonderauswertung der Repräsentativumfrage zum DGB-Index Gute Arbeit 2018, Sonderdruck für die IG Metall. Berlin.

Langhoff, T. (2005): ASTRA - Altersstrukturanalyse. In: GENERA - Neue Perspektiven für Ältere und Jüngere im Betrieb - Instrumente und Praxisbeispiele zur Bewältigung des demografischen Wandels. Düsseldorf.

Langhoff, T. (2009): Den demografischen Wandel im Unternehmen erfolgreich gestalten. Eine Zwischenbilanz aus arbeitswissenschaftlicher Sicht. Heidelberg.

Langhoff, T. \& Satzer, R. (2017): Gestaltung von Schichtarbeit in der Produktion, Working Paper Forschungsförderung Nr. 43, Juli 2017, hrsg. von der HansBöckler-Stiftung. Düsseldorf.

Langhoff, T., Bornewasser, M., Heidling, E., Kriegesmann, B. \& Falkenstein, M. (Hrsg.) (2015): Innovationskompetenz im demografischen Wandel. Konzepte und Lösungen für die unternehmerische Praxis. Wiesbaden.

Modis, T. (1998): Conquering uncertainty. New York.

Prezewowsky, M. (2007): Demografischer Wandel und Personalmanagement. Herausforderungen und Handlungsalternativen vor dem Hintergrund der Bevölkerungsentwicklung. Wiesbaden: Deutscher Universitäts-Verlag.

Reindl, J., Feller, C., Morschhäuser, M. \& Huber, A. (2004): Für immer jung? Wie Unternehmen des Maschinenbaus dem demographischen Wandel begegnen. Frankfurt.

Rosetti, K. \& Langhoff, T. (2015): Interne Potenziale. Kompetenzen von Mitarbeiterinnen und Mitarbeitern erkennen, nutzbar machen, entfalten. Dortmund.

Rump, J. \& Eilers, S. (Hrsg.) (2017): Auf dem Weg zur Arbeit 4.0, Innovationen in HR. Berlin/Heidelberg.

Szymanski, H. \& Lange, A. (2013): Den demografischen Wandel in der Eisen- und Stahlindustrie gestalten - eine Handlungshilfe zur alter(n)sgerechten Arbeitsgestaltung. Bochum.

Volkholz, V. \& Langhoff, T. (2008): Altern als Wettbewerbsfaktor, unveröffentlichtes Papier zum Forschungsbedarf im demografischen Wandel. Dortmund. 